\title{
Lipid emulsion treatment for ventricular tachycardia induced by the toxicity of multiple herbs
}

Ju-Tae Sohn ${ }^{1,2}$

'Department of Anesthesiology and Pain Medicine, Gyeongsang National University College of Medicine, Gyeongsang National University Hospital, Jinju, Korea

${ }^{2}$ Institute of Health Sciences, Gyeongsang National University, Jinju, Korea

\section{Dear Editor,}

It was with particular interest that I read the article by Han et al.," titled "Intravenous lipid emulsion therapy for cardiac arrest and refractory ventricular tachycardia due to multiple herb intoxication," that was recently published in Clinical and Experimental Emergency Medicine.' Our research group has previously reported that lipid emulsion can be effective in the treatment of local anesthetic systemic toxicity, as well as intractable cardiovascular collapse induced by nonlocal anesthetic drugs with high lipid solubility (log [octanol/water] partition coefficient: $>2$ ). ${ }^{2}$ I believe the following comments, based on the findings of our group and other researchers, would enable readers to gain a better understanding of the case report presented by Han et al.' First, a toxic dose of bupivacaine (log [octanol/water] partition coefficient: 3.41 ), a highly lipidsoluble amino-amide local anesthetic, causes conduction block and myocardial depression via the inhibition of cardiac sodium, potassium, and calcium channels, leading to QRS widening, ventricular tachyarrhythmia, and cardiac arrest. ${ }^{2}$ Similarly, it has been shown that the toxicity of non-local anesthetic drugs with high lipid solubility, such as imipramine, bupropion, flecainide, and diphenhydramine, causes QRS widening and cardiac arrhythmia via inhibition of cardiac sodium channels, which could be responsive to lipid emulsion treatment. ${ }^{3}$ Lipid emulsion attenuates the blockade of cardiac sodium channels by bupivacaine, and also has a direct positive inotropic effect. ${ }^{2}$ Thus, it may be effective in the treatment of ventricular tachycardia and myocardial depression induced by the suspected toxicity of multiple herbs described in the case report presented by Han et al. ' Second, although the "lipid sink theory" was initially one of the most widely accepted mechanisms explaining the effects of lipid emulsion treatment, ${ }^{2}$ the "lipid shuttle" concept, which states that the lipid phase of a lipid emulsion absorbs highly lipid-soluble local anesthetics such as bupivacaine from the heart, is now widely considered to be an indirect mechanism underlying the effect of lipid emulsion treatment. ${ }^{2}$ Accordingly, the lipid emulsion with bupivacaine partitioned in its lipid phase is transported to the liver and muscles for detoxification and storage, leading to enhanced redistribution. ${ }^{2}$ Third, Han et al. ${ }^{1}$ describe that extracorporeal membrane oxygenation was applied prior to the administration of lipid emulsion, and in this regard, it has previously been observed that administration of high-dose lipid emulsion to critically ill patients on extracorporeal membrane oxygenation can sometimes cause reduced circuit flow due to the viscosity of the lipid emulsion. ${ }^{4}$ Fourth, the triglyceride present in the SMOFlipid used has been shown to have a short plasma half-life ( $21.92 \pm 8.15$ minutes), whereas the plasma half-life of non-local anesthetic drugs (including amlodipine [36 hours] and bupro-
Received: 12 February 2020

Revised: 12 February 2020

Accepted: 19 March 2020

Correspondence to: Ju-Tae Sohn Department of Anesthesiology and Pain Medicine, Gyeongsang National University Hospital, 79 Gangnam-ro, Jinju 52727, Korea

E-mail: jtsohn@gnu.ac.kr

ORCID

https://orcid.org/0000-0003-0102-5800

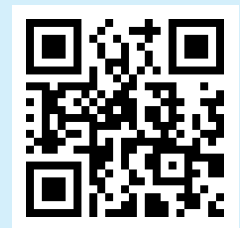

How to cite this article:

Sohn JT. Lipid emulsion treatment for ventricular tachycardia induced by the toxicity of multiple herbs. Clin Exp Emerg Med 2020;7(2):139-140.

This is an Open Access article distributed under the terms of the Creative Commons Attribution Non-Commercial License (https:// creativecommons.org/licenses/by-nc/4.0/). 
pion [ $29 \pm 9$ hours]), which can produce systemic toxicity, is significantly longer than that of the SMOFlipid triglyceride. ${ }^{5}$ If the continuous administration of SMOFlipid had been stopped earlier, the reduced drug level (amlodipine) and improved mental status (bupropion) observed in lipid emulsion treatment may have followed a rebound increase in drug level and deterioration in mental status, respectively, due to the short half-life of the SMOFlipid triglyceride. $^{5}$ Thus, from the perspective of systemic toxicity, the half-life of administered drugs should be taken into consideration in lipid emulsion treatment. Further studies regarding the dosage and timing of administration of lipid emulsions in the management of this toxicity are required owing to the longer period of absorption than that achieved via intravenous administration. I believe, however, that this report by Han et al. ${ }^{1}$ provides important data regarding the use of lipid emulsion as a non-specific antidote in the management of suspected multiple herb intoxication.

\section{CONFLICT OF INTEREST}

No potential conflict of interest relevant to this article was reported.

\section{REFERENCES}

1. Han S, Lim H, Noh H. Intravenous lipid emulsion therapy for cardiac arrest and refractory ventricular tachycardia due to multiple herb intoxication. Clin Exp Emerg Med 2019;6:366-9.

2. Ok SH, Hong JM, Lee SH, Sohn JT. Lipid emulsion for treating local anesthetic systemic toxicity. Int J Med Sci 2018;15:71322.

3. Bruccoleri RE, Burns MM. A literature review of the use of sodium bicarbonate for the treatment of QRS widening. J Med Toxicol 2016;12:121-9.

4. Sin JH, Tom A, Toyoda A, Roy N, Hayes BD. High-dose intravenous lipid emulsion affecting successful initiation of continuous venovenous hemofiltration and extracorporeal membrane oxygenation. Clin Toxicol (Phila) 2018;56:149-50.

5. Therapeutic Goods Administration. Australian public assessment report for soya oil, medium chain triglycerides, olive oil, fish oil [Internet]. Woden: Therapeutic Goods Administration; 2010 [cited 2020 Feb 11]. Available from: https://www.tga. gov.au/sites/default/files/auspar-smoflipid.pdf. 